\title{
Active Queue Management Techniques for Congestion Control in TCP Communication Networks: New Prospective
}

\author{
Keerthipati. Kumar, R.A. Karthika
}

\begin{abstract}
: the computer network area has grown very fast from previous years, as a result of which the control of traffic load in the network is at a higher priority. In network, congestion occurs if numbers of coming packets exceed, like bandwidth allocation along with buffer space. This might be due to poor network performance in terms of throughput, packet loss rate, and average packet queuing delay. For enhancing the overall performance when this network will become congested, numerous exclusive aqm (active queue management) techniques were proposed and few are discussed in this research paper. Particularly, aqm strategies are analyzed in detail as well as their obstacles along with strengths are emphasized. There are several algorithms which are under the aqm like ared, fred, choke, red (random early detection), blue, stochastic fair blue (sfb), random exponential marking (rem), svb, raq, etc.
\end{abstract}

Keywords: Congestion Control, AQM, RED (Random early detection), ARED, SFB (Stochastic Fair Blue), CHOKe.

\section{INTRODUCTION}

When the number of incoming packets is high contending for restricted shared resources, including outgoing bandwidth and queue buffer, congestion might additionally evident in the data communication. Throughout, congestion several packets revel in postpone or be dropped because of queue overloaded. Results of Congestion degrades the overall throughput as well as high loss rate of packets. Congestion also reduces reliability along with efficiency of the complete network, moreover, performance will also collapse at high traffic, and therefore no packets are delivered. At the internet traffic tends to range. Ideally, a router queue control set of rules must allow transient bursty traffic along with penalizing flows which consistently overuse bandwidth. Additionally, set of rules must save you excessive put off via restricting the queue duration, keep away from underutilization by using allocating temporary queueing, along giving sources pretty in one of a kind styles of site visitors [1].

Practically, most of the routers are arranged that used basic Drop Tail algorithm [2] as it is simple and easy to use having minimum computation overhead, however, gives unsatisfactory overall performance. In order to solve this specific hassle, several various queue management algorithms are there for example $\mathrm{CHOKe}$ (CHOose and Kill for unresponsive flows, CHOose and Keep for responsive flows,), SFB (Stochastic Fair BLUE), BLUE, FRED (Flow

Revised Manuscript Received on December 30, 2019.

Keerthipati. Kumar, Research Scholar, Computer Science And Engineering, Vistas, Chennai

R.A. Karthika, Associate Professor, Computer Science And Engineering, Vistas, Chennai
Random Early Drop), RED (Random Early Drop) [3, 4, 5, 6] Several algorithms state that they are able to offer truthful sharing amongst extraordinary flows without imposing an excessive amount of deployment complexity.

Mostly, these proposed algorithms just focus on one phase of an issue (either it is computational overhead, deployment complexity, or fairness), remove the limitations of lastly used algorithms as well as their simulations setting are distinct from one another. Therefore, all these make very difficult for evaluating as well as choosing one to utilize under specific traffic load.

\section{ACTIVE QUEUE MANAGEMENT}

In IP networks the important goal of AQM "Active Queue Management" was to balance the work of end-system protocols like in congestion control, TCP (Transmission Control Protocol) thus maximizing the utilization of network and decreasing the loss and packets delay. [2] AQM is a user that equally dividends bandwidth and manages congestion over the routers/internet. The subject on the congestion, metric effective queue management has listed load-based, queue-based length, load-based, and length-based.

\section{A. Queue length based AQM Algorithms:}

A QMs congestion based on Queue is measured through queue size as well as this movement is taken via keeping fixed of queues by means of Internet routers, one in line with interface, which keeps packets scheduled to begin extinct on the interface. In case the length of queue is shorter as compared to its higher restriction length, a packet is set on the queue and in any other case it will drop. It has one main limitation that packets' backlog is required inherently through the control mechanism when the congestion is discovered in queue.

\section{a. RED (Random Early Drop)}

"Sally Floyd and Van Jacobson [2] proposed a mechanism called Random Early Detection (RED) that aims at avoiding congestion". The work is inspired via an impartial of maintaining small average sizes of a queue in routers. This might be achieved by means of labeling or dropping certain packets that attain a positive threshold role inside the queue. It is possible to use ECN for marking packets suggesting congestion on the route.

RED keeps an EWMA (exponentially-weighted moving average) of the queue size that makes use of to find out congestion. 
If the duration dropped of common queue exceeds a minimal threshold (minth), randomly each packet is marked or an ECN (explicit congestion notification) bit [2]. If the size of common queue exceeds the most threshold (maxth), every packet is marked or dropped.

Whereas RED has many advantageous but it has also some limitations.

\section{Drawbacks of RED Algorithm:}

1. Fairness Problem: Generally expressed in 2 aspects: first is a fair connection of every TCP, sometime discard rate of packet is similar for every connection, although connection with smaller RTT bandwidth is not fair; second is TCP fairness flow with UDP flow, a packet is discarded as responding by TCP flows, however not by UDP flow. These outcomes will increase the bandwidth of UDP flows.

2. Priority Problem: Priority concept is the absence of the RED algorithm. Which is not suitable for Internet.

\section{b. Flow Random Early Drop (FRED)}

FRED [4] is a new version of RED that removes the hassle triggered with help of non-responsive flows as it utilized per-active go with the flow for making unique dropping or making probabilities link with distinct usages bandwidth. It entirely keeps track of passes which have packets in the buffer, thus the importance of FRED is proportional to the buffer dimension and free from the entire flow volumes. FRED utilized 2 major parameters: minq (minimum number of packets) along with maximum (maximum number of packets allowed to buffer every flow to FRED [6] and a global variable for finding the average of each active flow. A wide variety of active flows is maintained by it and every flow, FRED calculates multiple times if the flow is no longer responsive (maxq< qleni)

\section{Drawbacks of FRED:}

The "fundamental" FRED set of rules does not do very well while a massive range of (TCP) flows proportion a bottle neck link. If there's large wide variety of (TCP) flows, the queue inside the router can be close to its potential. A short burst of packet arrival will reason a queue overflow if queue is virtually full. When a queue over glide happens, the FRED algorithm degenerates into a drop tail.

\section{c. Adaptive Random Early Detection (ARED)}

ARED set of rules [7] plays whether or not to create greater or much less violent RED based on looking at the common period of the queue [8]. If the common period of the queue moves from side to side, early detection is excessively competitive. On the alternative hand, if the average period of the line movements from side to side, early detection is simply too traditionalist. The set of rules adjusts the chance based on how competitive it thinks visitors have been removed. Adapting the RED variable and setting the RED parameters routinely, thereby assist in retaining a steady average queue length as well as increasing the sensitivity of RED parameters. Nonetheless, ARED leaves the choice of goal queue duration to network operators who want to trade policies among use and delay.

\section{d. CHOKe}

The main objective of CHOKe [6] is to make its mechanism simple and easy when controlling an unresponsive flows. For achieving these objectives, small modifications are done on FCFS queue having RED AQM. Once a packet arrives, in case the size of queue is more as compared to minth; randomly a packet is drawn by $\mathrm{CHOKe}$ from buffer as well as it will compared with another arriving packet. In case they have similar flow, after that both the packets will drop, or in any other case the coming packet is prevalent in queue having a dropping chance as calculated by RED. The main concept of CHOKe is FIFO as it is apparently to possess packets that belong to unresponsive flows pretty the ones of responsive ones, thus they chose for comparison. Hence this is the main reason that mostly packets are dropped from unresponsive flows. CHOKe is fairly clean for implementing, controls unresponsive flows, and keeps minimal information mainly for $\mathrm{CHOKe}$ with more than one drop applicants.

\section{Drawbacks of choke:}

Only whilst several packets from those flows within the buffer on congestion time can $\mathrm{CHOKe}$ which manipulate unresponsive flows. This is because that CHOKe does not save the tune of these flows that are unresponsive. However, due to its probabilistic set of rules, few reactive flows might be unfairly punished.

There are many other queue lengths based algorithms are available such as FRED, SHRED, RARED, HRED, SRED, DSRED, PDRED, MRED, etc.

\section{B. Load based AQM Algorithms:}

\section{a. Blue}

Blue is basically an AQM algorithm that totally depends upon dropped packets along with link utilization. A variable $\mathrm{pm}$ is maintained by it for estimating the probability of dropping packets or marking packets. In case a queue is full that it automatically drop these packets, and $\mathrm{pm}$ is incremented through a $\mathrm{d} 1$ factor. In case a queue is empty and has no packet then it starts marking these packets and $\mathrm{pm}$ is reduced through $\mathrm{d} 2$ factor. The $\mathrm{d} 1$ value always set as that it is substantially large than $\mathrm{d} 2$. As link is underutilized whilst the congestion management is either conservative or competitive, but packet loss [9] happens best if the congestion mechanism is too conservative. It also uses another parameter known as freeze time that helps to determine the interval time among successive updates. It also permits the modifications inside marking the possibility to take effect earlier as compared to the value is updated once more.

\section{Drawbacks of Blue Algorithm:}

BLUE makes use of two hashing characteristics to discover the 2 non-responsive flows. If we have a big wide variety of non-responsive flows, they are able to harm the packing containers, and TCP flows may be confused for being non-responsive, ensuing in their unnecessary penalization. 
Another difficulty is that it is polluted forever once a flow is labelled. If the waft restrains itself in a while, BLUE will nonetheless try and lessen its sending rate by way of losing packets.

\section{b. SFB}

Stochastic Fair Blue (SFB) [10] is a new approach for managing unresponsive flows without utilizing queue occupancy schema. SFB scalable detects and fee-limits non-responsive flows by the usage of marking chance derived from the Blue queue control algorithm. SFB also helps to organize data structure in $\mathrm{L}$ hash tables of various hash functions having $\mathrm{N}$ items for every table [10]. Every item, that ia called as a bin, saves every item's time flow as well as its dropping or marking probability pm. Every arrived packet is going too hashed, utilizing few string forms like flow ID, in every L hash every item is counted and after that stored packet is increased by one in bin. In case the packet amount is larger in a bin as compared to certain number, packet will be dropped and bin is raised by few amounts. Conversely, pm reduced in case the number of packets drops to 0 in a bin. It is discovered that a non-responsive glide speedy drives Pm to one in all of the L containers it's far hashed into. The final dropping or marking probability is, although find out the minimal value of this pm. The selection of a packet depends upon Pmin the minimal Pm cost of complete bins. In case the value of Pminis 1, the packet is recognized as a non-responsive flow. There are several parameters that might be utilized within the SFB algorithm [10] for example Bin_Sizeis, Boxtime, Hinterval L, N, freeze_time, d2, d1, Bin Size, qlen, bin space. "Qlenis the actual queue duration of every bin. For every bin, d1, d2 and freeze_timehave the identical which means as that in Blue. Besides, $\mathrm{N}$ and $\mathrm{L}$ are associated with the dimensions of the accounting packing containers, for the boxes are organized in L tiers with $\mathrm{N}$ bins in each level. Hintervalis the time c program languageperiod used to alternate hashing functions in our implementation for the double buffered shifting hashing. Box time is utilized by penalty container of SFB as a time c language used to manipulate how a whole lot bandwidth those non-responsive flows ought to take from bottleneck links".

\section{Queue length and rate based AQM Algorithms:}

\section{a. REM}

The most critical intention of the REM algorithm is decoupling congestion degree from common overall performance degree [11]. The congestion level demonstrates additional need for account and bandwidth for the design of consumers, even though the performance measure indicates delay and queue length. REM is basically same as to RED (Random Early Detection) algorithm [1] within 2 vital aspects: (I) Unlike RED that utilizes widespread queue duration, REM uses a one-of-a-kind technique to amount congestion, along with (ii) REM calculates the drop or mark possibility in various other manners than RED [1].

\section{b. Stabilized Virtual Buffer (SVB)}

SVB (Stabilized Virtual Buffer)[12] is just like REM. In this set of rules for queue length and packet arrival rate stabilization unique from REM. It continues digital queue along with responds to dynamic visitors flows faster for better balance.

\section{c. $\operatorname{RaQ}$}

$\mathrm{RaQ}$ [13] makes use of the input charge and cutting-edge queue length for calculating the packet making/dropping possibility. From the control component concept, RaQ may be regarded as dual loop remarks manage. The internal loop of an algorithm is fee comments while the outer loop controls the queue duration. Therefore, fee remarks manage lets in $\mathrm{RaQ}$ to reply to congestion rapidly, in order to reduce the packet loss because of queue size as well as buffer overflow feedback manipulate stabilizes queue length of $\mathrm{RaQ}$. Hence, it is able to obtain predictable queuing lengthen and lower make bigger jitter.

\section{ACTIVE QUEUE MANAGEMENT COMPARISON}

The most important features of the algorithms have been summarized in the table

Table 1. Important futures of the AQM Algorithms

\begin{tabular}{|c|c|c|}
\hline $\begin{array}{c}\text { AQM } \\
\text { Schemes }\end{array}$ & $\begin{array}{c}\text { AQM } \\
\text { Algorithm }\end{array}$ & Important features \\
\hline \multirow[t]{3}{*}{$\begin{array}{l}\text { Queue } \\
\text { Based }\end{array}$} & RED & $\begin{array}{l}\text { It is used based on a } \\
\text { foundation for every } \\
\text { different algorithm cited } \\
\text { in the paper. It was } \\
\text { broadly utilized in } \\
\text { mixtures with TCP, as } \\
\text { well as have few } \\
\text { Drawbacks that showed } \\
\text { the result in the } \\
\text { progressed algorithms. }\end{array}$ \\
\hline & FRED & $\begin{array}{l}\text { FRED [4] is a new version } \\
\text { of RED that removes the } \\
\text { hassle triggered with help } \\
\text { of non-responsive flows as } \\
\text { it utilized per-active go } \\
\text { with the flow for making } \\
\text { unique dropping or } \\
\text { making probabilities link } \\
\text { with distinct usages } \\
\text { bandwidth. FRED utilized } \\
2 \text { major parameters: minq } \\
\text { (minimum number of } \\
\text { packets) along with } \\
\text { maximum (maximum } \\
\text { number of packets allowed } \\
\text { to buffer every flow to } \\
\text { FRED [6] and a global } \\
\text { variable for finding the } \\
\text { average of each active } \\
\text { flow. }\end{array}$ \\
\hline & ARED & 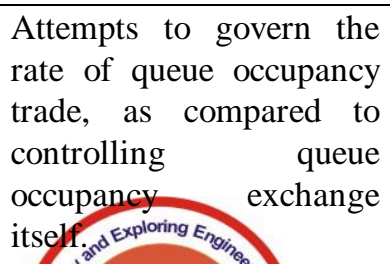 \\
\hline Blue Eyes In & on & \\
\hline
\end{tabular}




\begin{tabular}{|c|c|c|}
\hline & $\mathrm{CHOKe}$ & $\begin{array}{l}\text { Ensures that the queue } \\
\text { contents represent } \\
\text { 'enough statistics } \\
\text { approximately incoming } \\
\text { visitors as the queue is } \\
\text { more likely to include } \\
\text { packets from flows that } \\
\text { are misbehaved. This tries } \\
\text { to make these } \\
\text { misbehaving flows equal } \\
\text { with the aid of } \\
\text { discriminating against } \\
\text { them. }\end{array}$ \\
\hline \multirow[t]{2}{*}{ Rate Based } & BLUE & $\begin{array}{l}\text { Blue is basically an AQM } \\
\text { algorithm that totally } \\
\text { depends upon dropped } \\
\text { packets along with link } \\
\text { utilization. A variable pm } \\
\text { is maintained by it for } \\
\text { estimating the probability } \\
\text { of dropping packets or } \\
\text { marking packets. }\end{array}$ \\
\hline & SFB & $\begin{array}{l}\text { Attempts to make sure } \\
\text { equity through figuring } \\
\text { out and rate-proscribing } \\
\text { non-responsive flows } \\
\text { based on accounting } \\
\text { mechanisms which might } \\
\text { be utilized in BLUE also. } \\
\text { Every arrived packet is } \\
\text { going too hashed, } \\
\text { utilizing few string forms } \\
\text { like flow ID, in every L } \\
\text { hash every item is counted } \\
\text { and after that stored } \\
\text { packet is increased by one } \\
\text { in bin. }\end{array}$ \\
\hline \multirow[t]{3}{*}{$\begin{array}{c}\text { Queue } \\
\text { based } \\
\& \\
\text { Rate Based }\end{array}$} & REM & $\begin{array}{l}\text { Steady the input rate and } \\
\text { queue irrespective of a } \\
\text { number of sources sharing } \\
\text { link and seek average link } \\
\text { prices as a congestion } \\
\text { measure and alert the } \\
\text { source by end-to-end } \\
\text { labeling probability in } \\
\text { order to take the rate. }\end{array}$ \\
\hline & SVB & $\begin{array}{l}\text { In SVB the incoming } \\
\text { packets are labelled with } \\
\text { an opportunity primarily } \\
\text { depends upon virtual } \\
\text { queue occupancy along } \\
\text { with existing virtual } \\
\text { buffer }\end{array}$ \\
\hline & $\mathrm{RaQ}$ & $\begin{array}{lr}\mathrm{RaQ} & \text { Evaluation } \\
\text { demonstrated the RaQ } \\
\text { prevalence in packet loss, } \\
\text { attaining } & \text { faster }\end{array}$ \\
\hline
\end{tabular}

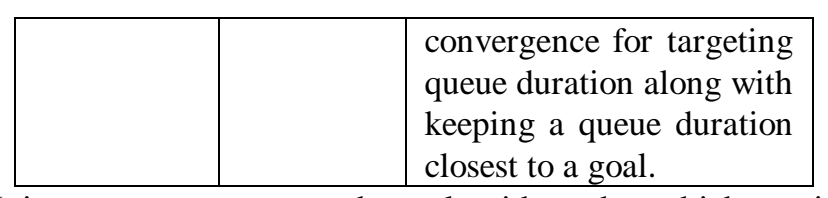

It is not easy to compare these algorithms that which one is good or which one is bad with every element, specifically for deployment complexity. Therefore, the essential traits that might be positioned are: Some of these algorithms provide immoderate hyperlink usage, Blue and RED don't select out as well as penalize non-responsive waft, whereas the opposite algorithms preserve honest sharing amongst certainly one of a kind traffic flows, the equity is achieved the usage of wonderful methods, FRED document according to-active-drift data, SFB statistically multiplex buffers to packing containers, however wants to reconfigured with non-responsive flows pattern, with coming packets whole algorithms has computation overhead, their vicinity necessities are special. Our assessment outcomes are summarized in the below table:

\section{Table 2. Comparison of AQM Algorithm}

\begin{tabular}{|c|c|c|c|c|c|c|c|}
\hline $\begin{array}{c}\mathrm{AQM} \\
\text { Schemes }\end{array}$ & $\begin{array}{c}\mathrm{AQM} \\
\text { Algorith } \\
\mathrm{m}\end{array}$ & Fairness & $\begin{array}{c}\text { Computatio } \\
\mathrm{n}, \\
\text { Complexity }\end{array}$ & $\begin{array}{c}\text { Throughpu } \\
\mathrm{t}\end{array}$ & $\begin{array}{c}\text { Link } \\
\text { Utilization }\end{array}$ & $\begin{array}{l}\text { Loss } \\
\text { Rate }\end{array}$ & $\begin{array}{l}\text { Queue } \\
\text { Stability }\end{array}$ \\
\hline \multirow{4}{*}{$\begin{array}{l}\text { Queue } \\
\text { Based }\end{array}$} & RED & Low & High & Low & High & High & Moderate \\
\hline & FRED & High & Very High & High & High & Low & Moderate \\
\hline & ARED & Low & High & Moderate & High & $\begin{array}{c}\text { Moderat } \\
e\end{array}$ & High \\
\hline & CHOKe & $\begin{array}{c}\text { Moderat } \\
\mathrm{e}\end{array}$ & Moderate & Moderate & Moderate & $\begin{array}{c}\text { Moderat } \\
\mathrm{e}\end{array}$ & Moderate \\
\hline \multirow[t]{2}{*}{ Rate Based } & BLUE & Low & Moderate & Very High & High & $\begin{array}{c}\text { Moderat } \\
\mathrm{e}\end{array}$ & Low \\
\hline & SFB & $\begin{array}{c}\text { Moderat } \\
\mathrm{e}\end{array}$ & High & Moderate & High & $\begin{array}{c}\text { Moderat } \\
\mathrm{e}\end{array}$ & Moderate \\
\hline Queue & REM & Low & High & Very Low & High & Low & Very Low \\
\hline based & SVB & Low & High & Low & High & Low & Very Low \\
\hline $\begin{array}{c}\& \\
\text { Rate based }\end{array}$ & $\mathrm{RaQ}$ & Low & High & Low & High & Low & $\begin{array}{l}\text { Very } \\
\text { Low }\end{array}$ \\
\hline
\end{tabular}

\section{CONCLUSION AND FUTURE WORK}

In this research paper, a survey is carried out on AQM (Active Queue Management) mechanisms. We have explained the main goals of AQM. The AQM quality schemes were evaluated in this study, chosen from among the several published over previous years. These algorithms are compared for RED, FRED, ARED, CHOKe, BLUE, SFB, REM, SVB, and RaQ. It has been determined that the RED AQM set of rules does not adjust the queue length after testing and regulates the queue width. BLUE AQM algorithm significantly reduces the buffer requirement necessary to support differentiation of the material. FRED AQM set of algorithms records efficient float information. Statistically multiplex SFB buffer to packing containers, although it required to be reconfigured with a big range of non-responsive flows. By hybridizing RED, SFB, BLUE and Choke AQM algorithms, we meant to expand a new algorithm that permits the brand new set of rules to take gain of current algorithms and deliver a better end result. 


\section{REFERENCES}

1. V. Jacobson. Congestion avoidance and control. Proceedings ofSIGCOMM 88, pages 314-329, 1988

2. D. Sanghi and A. Agrawala, "DTP: An Efficient Transport Protocol", University of Maryland tech report UMIACS-TR91-133, October 1991.

3. S. Floyd and V. Jacobson, Random early detection gateways for congestion avoidance, IEEE/ACM Transactions on Networking, Vol.1, 397-413,1993.

4. D.Lin., R.Morris.," Dynamics of Random early Detection”, Proceedings of ACM SIGCOMM,Octobet 1997

5. Wu -Chung Feng., Kang G.Shin., Dilip D.Kandlur., Debanjan Saha ., "The BLUE active queue management algorithms", IEEE ACM Transactions on Networking, Volume 10 Issue 4, pp.513-528, August 2002.

6. Pan ,R., Parbhakar, B., and Psounis K., "CHOKe, a stateless active queue management scheme for approximating fair bandwidth allocation", Proceedings of IEEE INFOCOM Conference on Computer Communications, pp.942-951, March2001.

7. Yue-Dong Xu, Zhen-Yu Wang. Hua Wang., “ARED: A Novel Adaptive Congestion Controller" IEEE International Conference on Machine Learning and Cybernetics, August 2005.

8. Mark Parris, Kevin Jeffay ,F. Donelson Smith "Lightweight Active Router-Queue Management for Multimedia Networking” Multimedia Computing and Networking 1999, Proceedings, SPIE Proceedings Series, Volume 3654, San Jose, CA, January 1999, pages 162-174.

9. Wu-chang Feng; Dilip D. Kandlur; Debanjan Saha; Kang G. Shin (April 1999). "BLUE: A New Class of Active Queue Management Algorithms" (PDF). Computer Science Technical Report. University of Michigan (CSE-TR-387-99). Retrieved June 8, 2013.

10. Wu-Chung Feng., Kang G.Shin., Dilip D.Kandlur., Debanjan Saha ", "The BLUE Active Queue Management Algorithms" IEEE ACM Transactions on Networking, August 2002.

11. Sanjeewa Athuraliya. REM: Active Queue Management. IEEE network 15.3, 3:48-53, 2001.

12. Xidong Deng., Sungwon Yi., George Kesidis., Chita R.Das., " Stabilised Virtual Buffer (SVB)-An active queue management scheme for internet quality of service", IEEE Globecom November 2002.

13. Jinsheng Sun., Moshe Zukerman ., " RaQ: a robust active queue management scheme based on rate and queue length", Computer Communications, Volume 30 Issue 8, PP. 1731-1741, June 2007 